\title{
RNAstructure: software for RNA secondary structure prediction and analysis
}

\author{
Jessica S Reuter, David H Mathews
}

\begin{abstract}
Background: To understand an RNA sequence's mechanism of action, the structure must be known. Furthermore, target RNA structure is an important consideration in the design of small interfering RNAs and antisense DNA oligonucleotides. RNA secondary structure prediction, using thermodynamics, can be used to develop hypotheses about the structure of an RNA sequence.

Results: RNAstructure is a software package for RNA secondary structure prediction and analysis. It uses thermodynamics and utilizes the most recent set of nearest neighbor parameters from the Turner group. It includes methods for secondary structure prediction (using several algorithms), prediction of base pair probabilities, bimolecular structure prediction, and prediction of a structure common to two sequences. This contribution describes new extensions to the package, including a library of $\mathrm{C}++$ classes for incorporation into other programs, a user-friendly graphical user interface written in JAVA, and new Unix-style text interfaces. The original graphical user interface for Microsoft Windows is still maintained.

Conclusion: The extensions to RNAstructure serve to make RNA secondary structure prediction user-friendly. The package is available for download from the Mathews lab homepage at http://rna.urmc.rochester.edu/RNAstructure. html.
\end{abstract}

\section{Background}

The prediction of RNA structure has received increasing attention over the last decade as the number of known functional RNA sequences, called non-coding RNA (ncRNA), has increased [1]. These new ncRNA sequences range in size from microRNAs to Xist [2,3]. They serve numerous roles, from modulating gene expression [4-6] to catalyzing reactions $[7,8]$.

One of the first steps to understanding the mechanism of action of an RNA is to determine its structure [9]. Secondary structure, defined as the set of canonical base pairs (AU, GC, and GU), can be determined using comparative analysis if a large number of sequences are available $[10,11]$. In comparative analysis, base pairs are determined when they are conserved in multiple sequences and instances of compensating base pair changes occur. Compensating base pair changes demonstrate the conservation of structure in spite of sequence

\footnotetext{
* Correspondence: David_Mathews@urmc.rochester.edu
Department of Biochemistry \& Biophysics and Center for RNA Biology,
University of Rochester Medical Center, 601 Elmwood Avenue, Box 712,

* Correspondence: David_Mathews@urmc.rochester.edu
Department of Biochemistry \& Biophysics and Center for RNA Biology,
University of Rochester Medical Center, 601 Elmwood Avenue, Box 712,

* Correspondence: David_Mathews@urmc.rochester.edu
Department of Biochemistry \& Biophysics and Center for RNA Biology,
University of Rochester Medical Center, 601 Elmwood Avenue, Box 712, Rochester, NY 14642, USA
}

(c) 2010 Reuter and Mathews; licensee BioMed Central Ltd. This is an Open Access article distributed under the terms of the Creative Commons Attribution License (http://creativecommons.org/licenses/by/2.0), which permits unrestricted use, distribution, and reproduction in any medium, provided the original work is properly cited. sequence being replaced by a homologous $\mathrm{AU}$ pair in another sequence. Comparative analysis, however, requires both significant user input and a large number of homologous sequences that can be aligned.

As an alternative to comparative analysis, the secondary structure of an RNA can be predicted for a single sequence using thermodynamics [9]. The thermodynamic methods are based on nearest neighbor rules that predict the stability of a structure as quantified by folding free energy change [12-14]. Often, structure prediction is accomplished by finding the lowest free energy structure, which is the single most probable structure in a folding ensemble [15]. Alternatively, structures can be sampled from the Boltzmann ensemble and a centroid, i.e. representative structure, determined [16,17]. Another alternative method for structure prediction is the prediction of a structure with the highest sum of pairing probabilities, called the maximum expected accuracy structure $[18,19]$.

Single sequence secondary structure prediction is reasonably accurate. On average, for sequences of fewer 
than 700 nucleotides, the accuracy of predicting known base pairs is as high as 73\% [14]. The accuracy, however, benchmarked lower when longer sequences were included [20,21]. Additional sources of information can be used to improve accuracy. For example, base pair probabilities can be determined using a partition function and highly probable pairs are more likely to be correctly predicted pairs [22]. Alternatively, using two or more homologous sequences to determine a conserved structure can result in significantly more accurate structure prediction [23-27]. Experimental data, such as enzymatic cleavage [13], chemical mapping [14], oligonucleotide array binding [28], SHAPE [29], and NMR data [30] can all be used to improve structure prediction accuracy.

In addition to structure prediction, the thermodynamic methods can be applied to other problems. For example, antisense oligonucleotide and siRNA design can be improved using thermodynamic predictions of self-structure in the oligonucleotides and target [31-36]. Sequences can be designed to fold to a specific structure [37,38]. Reverse-PCR primers can be designed to avoid self structure in the template that could prevent hybridization [39]. Novel types of ncRNAs can be found in genomes on the basis of folding stability [40-42].

In this contribution, the RNAstructure software package is described. RNAstructure first appeared in the literature in 1998 as a secondary structure prediction package [43]. At that time, it contained a method to predict the lowest free energy structure and a set of low free energy structures $[44,45]$. It was subsequently expanded to include bimolecular folding and hybridization thermodynamics with OligoWalk $[13,31,33]$. It was then expanded to include an algorithm for finding lowest free energy structures common to two sequences, Dynalign [23,41,46]; a partition function algorithm [22]; an alternative prediction method that can determine all low free energy structures for a sequence $[28,47]$; and stochastic sampling of structures [48]. It provides methods for constraining structures with enzymatic data [13], chemical mapping data [14], SHAPE [29], and NMR data [30]. Finally, recent extensions include PARTS [24], which calculates partition functions for secondary structures common to two sequences and can perform stochastic sampling of common structures [48]; MaxExpect, which finds maximum expected accuracy structures [18]; and a method for removal of pseudoknots, leaving behind the lowest free energy pseudoknot-free structure [49]. Several tutorials exist for using RNAstructure [50-52].

RNAstructure has been publicly available with a userfriendly interface for Microsoft Windows. Testing and development occur in-house using Unix/Linux text- based interfaces, but these have generally been available only upon request. The package is coded in $\mathrm{C}++$. Extensive benchmarks of individual components have been published [14,18,22-24,29-31,46,48,53,54].

Here, three major extensions of RNAstructure are reported. First, a new JAVA-based graphical interface (GUI) is available. This interface functions cross-platform and binaries are available for Apple OS-X and Linux. Second, text interfaces are now available for each component of the package. These interfaces use a standard Unix syntax and include online help. Binaries for the text interfaces are available for Microsoft Windows and source code, including Makefiles, are available for download. Finally, a new class library is available for programmers who want to incorporate the RNAstructure functions into $\mathrm{C}++$ programs. Each of these components is available for download and covered by the GNU Public License, version 2.

\section{Implementation}

\section{$\mathrm{C}++$ class library}

The $\mathrm{C}++$ class library encapsulates the $\mathrm{I} / \mathrm{O}$ functions of RNAstructure and also the secondary structure prediction and analysis methods. Four main classes are provided for accessing these functions: RNA, for single sequence structure predictions; Dynalign_object, for Dynalign calculations [23]; HybridRNA, for bimolecular structure prediction [33]; and Oligowalk_object, for OligoWalk [31,33] and OligoScreen [55] calculations. Two other notable classes are utilized by inheritance by the four main classes. Thermodynamics is a class that handles reading and storage of the nearest neighbor parameters. TwoRNA is a class that contains two RNA classes and is inherited by Dynalign_object and instantiated by HybridRNA. A class inheritance diagram is provided as Figure 1.

The classes are designed to be easily included in $\mathrm{C}++$ projects. The classes are compiled to Linux/Unix shared libraries, Windows dynamic link libraries, or Macintosh dynamic shared libraries. An included Makefile provides facility for this. Furthermore, the header (* ${ }^{*}$ h) files are commented using Doxygen-formatted comments that facilitate the creation of a manual http://www.stack.nl/ $\sim$ dimitri/doxygen/. Programmers can either compile their own manuals into html or latex using Doxygen or they can refer to precompiled html manuals included in the source code and posted on the RNAstructure website.

\section{Text interfaces}

The new text interfaces are designed to provide the features of RNAstructure for use on the command line and in scripts. Most programs available in the graphical user interfaces are provided with text interfaces. Each of 


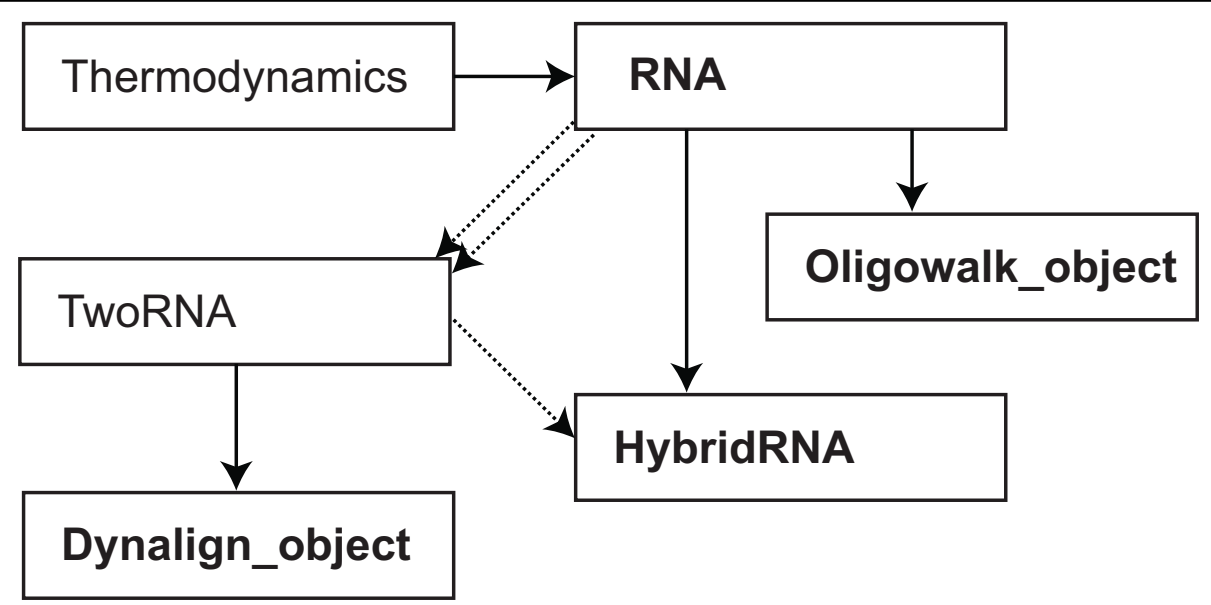

Figure 1 Class inheritance diagram. The four main classes that provide functionality, named in boldface, are RNA, Oligowalk_object, HybridRNA, and Dynalign_object. Solid lines show inheritance and the dotted lines indicate that a class contains an instance of another. TwoRNA contains two instances of RNA and HybridRNA contains an instance of TwoRNA.

these programs provides a brief description of the parameters when invoked without parameters. Additionally, invoking most programs with "-h," "-H," or "-help" will return a more detailed description of the parameters.

The text interfaces are built with ANSI-standard C++. Makefiles are included for compiling the programs in a Unix/Linux/OS X environment. Binaries for Microsoft Windows are also available for download.

The RNAstructure class library is used to implement most of the functions in the text interfaces. Therefore, the text interfaces can be used as tutorials by programmers implementing the RNAstructure algorithms in their own programs.

\section{JAVA Graphical User Interface}

The new JAVA GUI is a cross-platform re-implementation of the RNAstructure Windows GUI. This is designed to be user-friendly to make these algorithms accessible to a large audience. Executables are available for Apple OS-X and both 32-bit and 64-bit Linux. A Makefile is provided to facilitate local compilation on Unix/Linux environments.

The JAVA GUI utilizes the new RNAstructure $\mathrm{C}++$ class library to implement the algorithms. The connection between the JAVA front end and the $\mathrm{C}++$ back end is made using SWIG http://www.swig.org/. SWIG wraps the $\mathrm{C}++$ classes, making them accessible to JAVA. The SWIG-wrapped code is made available as part of the RNAstructure code download, so that the JAVA interface can be built on local machines that do not have SWIG installed.

\section{Availability of algorithms}

Table 1 shows the availability of the different singlesequence structure prediction and analysis algorithms in RNAstructure. It shows the names of the text interface programs, the corresponding function name and class library, and the menu name in the JAVA and Windows GUIs. Similarly, Table 2 shows the corresponding information for the multi-sequence methods (either for common secondary structure prediction or prediction of nucleic acid hybridization).

\section{Thermodynamic parameters}

The algorithms implemented in RNAstructure use nearest neighbor parameters to predict the stability of secondary structures. These include both free energy change parameters at $37^{\circ} \mathrm{C}$ and enthalpy change parameters to allow prediction of conformation stability at an arbitrary temperature. For RNA, these parameters are those most recent parameters from the Turner group $[12,14,54]$. For DNA, the parameters are derived from the experimental literature [56-92]. Most algorithms for RNA structure prediction can be invoked for DNA structure prediction using the class, text interfaces, or GUIs. DNA-RNA hybridization parameters are also used by OligoWalk [93].

\section{Unit testing}

The RNAstructure package now includes a facility for unit testing. A Makefile automates testing of the text interfaces and comparison with calculation standards. The same tests can be used to test the GUIs, but this 
Table 1 Single Sequence Methods

\begin{tabular}{|c|c|c|c|}
\hline Feature: & $\begin{array}{l}\text { Text Interface } \\
\text { Program: }\end{array}$ & $\begin{array}{l}\text { JAVA/Windows } \\
\text { GUI Menu Item: }\end{array}$ & $\begin{array}{l}\text { Class Library and } \\
\text { Function Name: }\end{array}$ \\
\hline Free energy minimization structure prediction [14] & Fold & $\begin{array}{l}\text { Fold RNA } \\
\text { Single Strand }\end{array}$ & RNA.:FoldSingleStrand \\
\hline Maximum expected accuracy structure prediction [18] & MaxExpect & $\begin{array}{l}\text { Predict } \\
\text { Maximum } \\
\text { Expected } \\
\text { Accuracy } \\
\text { Structure }\end{array}$ & RNA:MaximizeExpectedAccuracy \\
\hline Partition function [22] & partition & $\begin{array}{l}\text { Partition } \\
\text { Function RNA }\end{array}$ & RNA::PartitionFunction \\
\hline Efn2 (energy calculator) [13] & efn2 & Efn2 RNA & RNA:: CalculateFreeEnergy \\
\hline $\begin{array}{l}\text { Free energy minimization and generation of all suboptimal } \\
\text { structures }[28,47]\end{array}$ & Allsub & $\begin{array}{l}\text { Generate All } \\
\text { Suboptimal } \\
\text { RNA Structures }\end{array}$ & RNA::GenerateAllSuboptimalStructures \\
\hline Stochastic sampling of structures [17] & stochastic & $\begin{array}{l}\text { Stochastic RNA } \\
\text { Sampling }\end{array}$ & RNA::Stochastic \\
\hline Remove Pseudoknots [49] & RemovePseudoknots & $\begin{array}{l}\text { Break } \\
\text { Pseudoknots }\end{array}$ & RNA::BreakPeudoknots \\
\hline $\begin{array}{l}\text { Prediction of structures with pairs above specified pairing } \\
\text { probability threshold [22] }\end{array}$ & ProbablePair & $\begin{array}{l}\text { Output Probable } \\
\text { Structure }\end{array}$ & RNA::PredictProbablePairs \\
\hline Drawing secondary structure diagrams & draw & Draw & RNA::DetermineDrawingCoordinates \\
\hline NAPSS [30] & NAPSS & - & - \\
\hline
\end{tabular}

Table 2 Multiple Sequence Methods

\begin{tabular}{llll}
\hline Feature: & $\begin{array}{l}\text { Text Interface } \\
\text { Program: }\end{array}$ & $\begin{array}{l}\text { JAVA/Windows } \\
\text { GUI Menu Item: }\end{array}$ & $\begin{array}{l}\text { Class Library and } \\
\text { Function Name: }\end{array}$ \\
\hline Dynalign $[23,41,46]$ & dynalign & Dynalign RNA & Dynalign_object::Dynalign \\
\hline OligoWalk [31-33] & OligoWalk & OligoWalk & Oligowalk_object::Oligowalk \\
\hline OligoScreen [55] & oligoscreen & OligoScreen & Oligowalk_object::OligoScreen \\
\hline $\begin{array}{l}\text { Bimolecular structure prediction with intramolecular } \\
\text { pairs [33] }\end{array}$ & bifold & Fold RNA & HybridRNA::FoldBimolecular \\
\hline PARTS [24,48] & PARTS & - & \\
\hline $\begin{array}{l}\text { Bimolecular partition function (no intramolecular pairs) } \\
\text { [31] }\end{array}$ & bipartition & Partition & HybridRNA::PartitionFunctionBimolecular \\
& & Function RNA & \\
\hline $\begin{array}{l}\text { Bimolecular structure prediction without intramolecular } \\
\text { pairs }\end{array}$ & DuplexFold & - & HybridRNA::FoldDuplex \\
\hline
\end{tabular}

requires manual selection of the input. Unit testing is helpful when changes are made to the algorithms to ensure that structure prediction is unchanged. Unit testing is also important when RNAstructure is installed in environments that have not been previously tested, to ensure that the installation worked correctly.

\section{Results}

To demonstrate the utility of RNAstructure, an example of secondary structure prediction is provided, showing the input and output. The method utilized is free energy minimization and the example sequence is the $5 \mathrm{~S}$ rRNA from Pneumocystis carinii [94].
Figure 2 shows a screenshot of the "Fold RNA Single Strand" input form from the JAVA GUI. This program predicts the lowest free energy structure for an input sequence and a set of low free energy structures called suboptimal structures. The name of the input sequence is already selected in this screenshot by having clicked the "Sequence File" button. The default output (CT) file and the default parameters for determining suboptimal structures were determined by the program. Save files can be written to accelerate the prediction of an alternative set of suboptimal structures with a subsequent calculation. This view of the GUI shows the checkbox unchecked and so no save file will be written. The three 


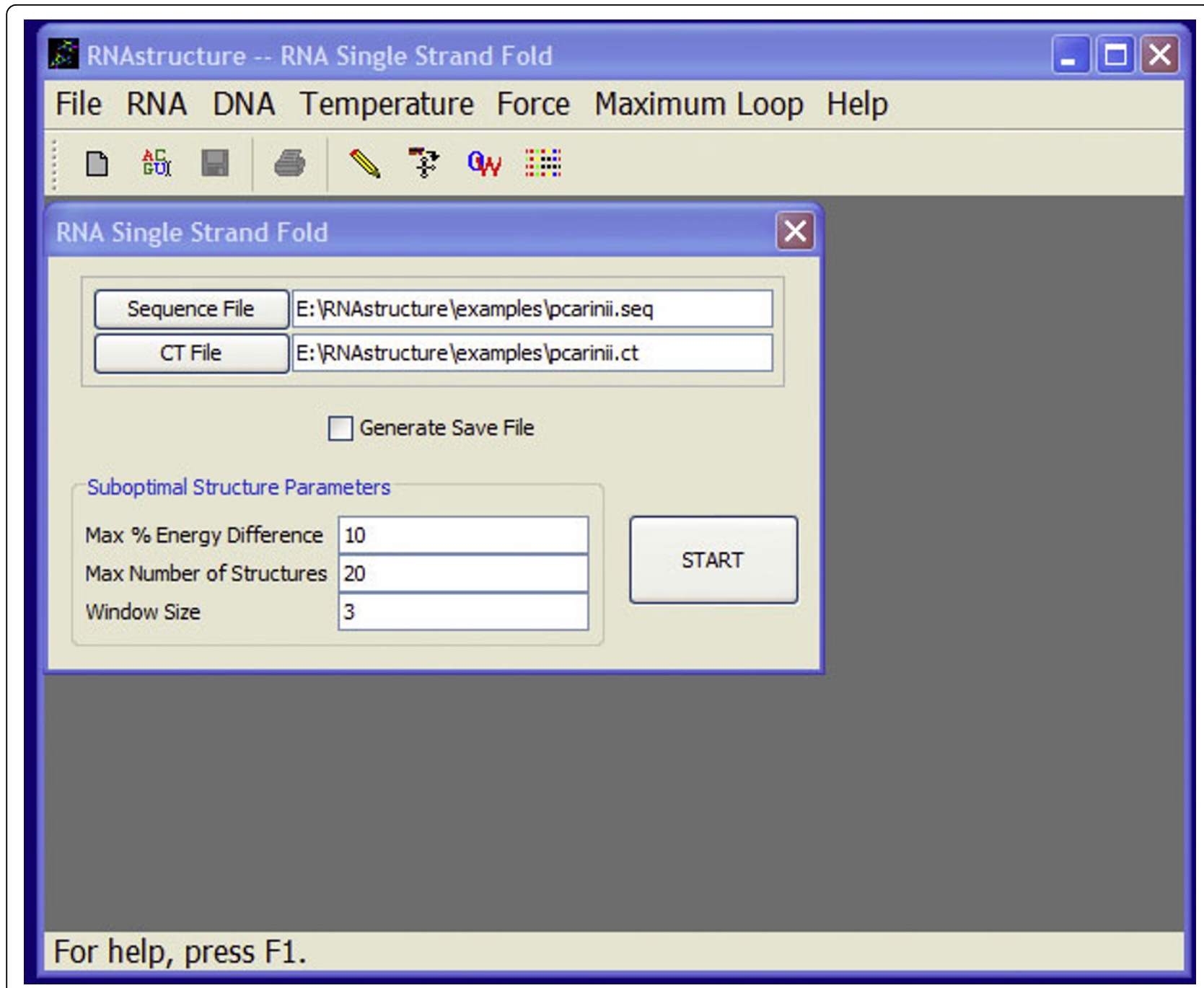

Figure 2 A screenshot of the JAVA GUI for predicting the lowest free energy structure for sequence "pcarinii.seq".

remaining parameters control the prediction of suboptimal structures. "Max \% Energy Difference" and "Max Number of Structures" place limits on the number of suboptimal structures predicted, with a maximum of the percentage difference in free energy change above the lowest free energy structure or an absolute limit on the number of structures, respectively. Suboptimal structures will be generated until the percent energy difference above the lowest free energy structure is reached, $10 \%$ in this case, or 20 structures have been generated. "Window Size" determines how different the suboptimal structures must be from each other in the set [44]. Zero places no restriction and larger integers become increasingly more stringent in the number of different pairs required between structures.

After clicking "Start," the calculation proceeds and the structure is then drawn as shown in Figure 3. The lowest free energy structure is shown by default and the user can display other structures by selecting the "Draw" menu item or by typing control-up-arrow or controldown-arrow.

The same calculation could have been performed on the command line using the program "Fold." The command line for the same default method is "Fold pcarinii. seq pcarinii.ct." A set of postscript images of the predicted structure can then be rendered using "draw pcarinii.ct pcarinii.ps."

Behind the scenes, the structure prediction is performed using the RNA class. For this example, the class was instantiated using a constructor that reads sequences: RNA::RNA("pcarinii.seq", 2, true). The integer 2 indicates that the file is a sequence file and the bool true indicates that the sequence is RNA (as opposed to DNA). Structure prediction is then 


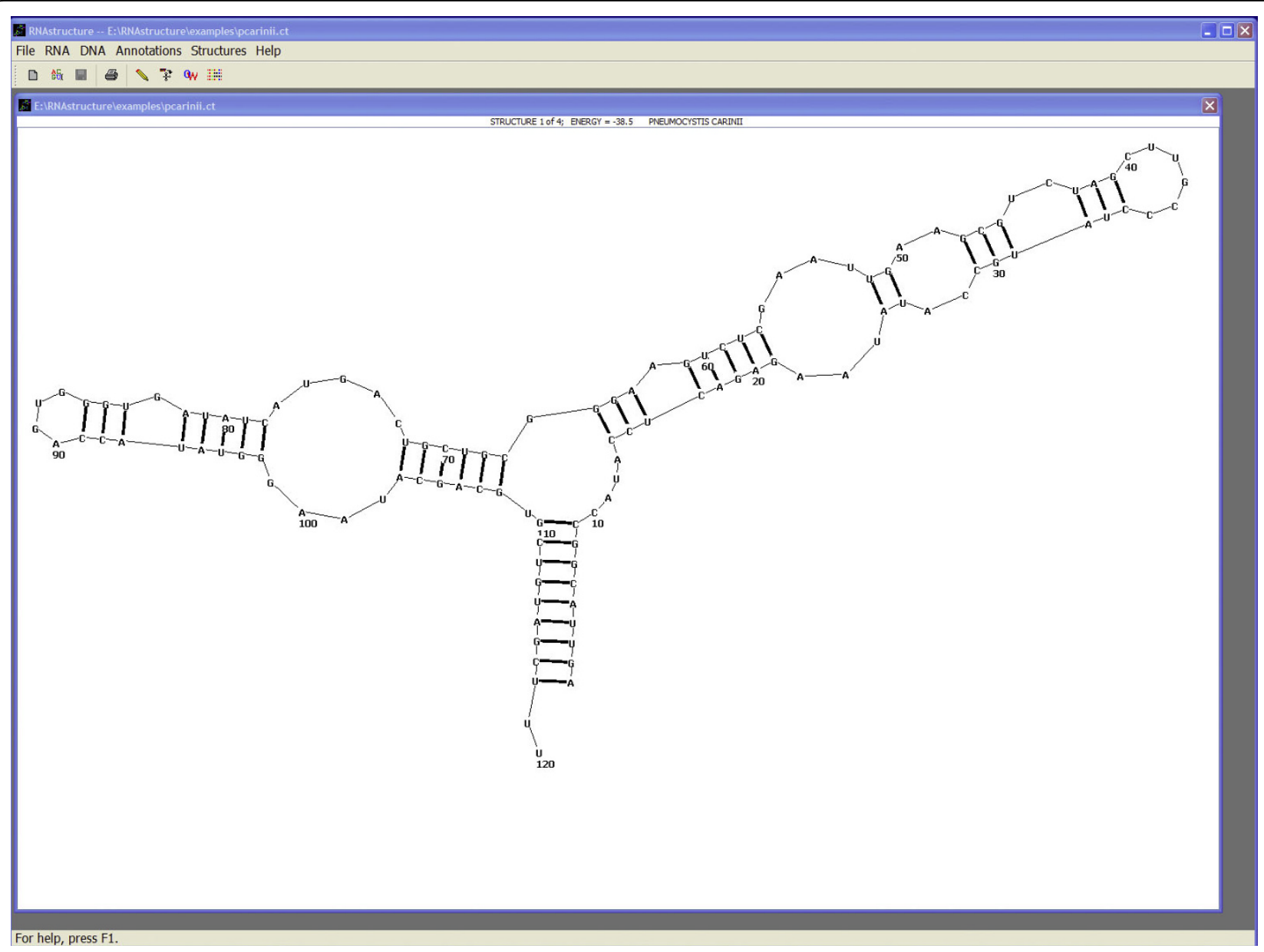

Figure 3 A screenshot of the JAVA GUI displaying the predicted lowest free energy structure for the sequence input as shown in Figure 2.

accomplished using RNA::FoldSingleStrand(10, 20, 3), where the parameters that control suboptimal prediction are the Max \% Energy Difference, the Max number of structures, and the Window Size, respectively, a appeared in the GUI (Figure 2). The pairing can then be queried using RNA::GetPair or the drawing coordinates can be determined using RNA:: DetermineDrawCoordinates.

\section{Conclusions}

RNAstructure is a software package for RNA secondary structure prediction and analysis. It is designed to make algorithms accessible for a variety of user needs. Userfriendly GUIs are available for Windows, using native Windows code, and for Linux/Unix and Macintosh OS-X using JAVA. Text interfaces are provided for performing calculations on the command line or for scripting. Finally, a $\mathrm{C}++$ class library is available to implement the algorithms into new programs. The package can be downloaded at http://rna.urmc.rochester.edu/RNAstructure.html.

\section{Availability and requirements}

- Project name: RNAstructure, version 5.0 and later

- Project home page: http://rna.urmc.rochester.edu/ RNAstructure.html

- Operating system(s):

Text interfaces: Compilation is platform independent. Executables are provided for Microsoft Windows and for 32- and 64-bit Linux.

Class library: Compilation is platform independent. Windows GUI: Windows XP or later.

JAVA GUI: Compilation is platform independent, but requires Sun JDK 1.6 or higher. Executables are provided for Macintosh OS-X (version 10.5 or later) and Linux. 


\section{- Programming language:}

Text interfaces, Class library, Windows GUI: C++ JAVA GUI: JAVA

\section{- Other requirements:}

Windows GUI: Compilation requires Microsoft Foundation Classes (MFC) as found in Microsoft Visual Studio 2005 or later and the Intel C++ compiler.

JAVA GUI: Requires Sun JAVA JDK version 1.6 or later. Recompilation of SWIG interface requires SWIG version 1.3 .39 or later.

\section{- License: GNU GPL}

- Any restrictions to use by non-academics: None.

\section{Abbreviations}

GUl: graphical user interface; ncRNA: non-coding RNA; siRNA: Small interfering RNA

\section{Acknowledgements}

This work was supported by National Institutes of Health grant R01GM076485 to D.H.M. The authors thank those who contributed to the code base.

\section{Authors' contributions}

JSR wrote the JAVA interface and the new text interfaces. DHM wrote the C ++ class library. Both authors contributed to the debugging of the code. DHM drafted the manuscript and both authors contributed to revisions.

Received: 23 October 2009 Accepted: 15 March 2010

Published: 15 March 2010

\section{References}

1. Eddy SR: Non-coding RNA genes and the modern RNA world. Nature Reviews 2001, 2:919-929.

2. Mello CC, Conte D Jr: Revealing the world of RNA interference. Nature 2004, 431(7006):338-342

3. Chow J, Heard $\mathrm{E}: \mathrm{X}$ inactivation and the complexities of silencing a sex chromosome. Curr Opin Cell Biol 2009, 21(3):359-366.

4. Wu L, Belasco JG: Let me count the ways: mechanisms of gene regulation by miRNAs and siRNAs. Mol Cell 2008, 29(1):1-7.

5. Storz G, Gottesman S: Versatile Roles of Small RNA Regulators in Bacteria. The RNA World Cold Spring Harbor: Cold Spring Harbor Laboratory PressGesteland RF, Cech TR, Atkins JF, third 2006, 567-594.

6. Tucker BJ, Breaker RR: Riboswitches as versatile gene control elements. Curr Opin Struct Biol 2005, 15:342-348.

7. Rodnina MV, Beringer M, Wintermeyer W: How ribosomes make peptide bonds. Trends Biochem Sci 2007, 32(1):20-26.

8. Doudna J, Cech T: The chemical repertoire of natural ribozymes. Nature 2002, 418:222-228.

9. Mathews $\mathrm{DH}$, Turner DH: Prediction of RNA secondary structure by free energy minimization. Curr Opin Struct Biol 2006, 16(3):270-278.

10. Gutell RR, Lee JC, Cannone JJ: The accuracy of ribosomal RNA comparative structure models. Curr Opin Struct Biol 2002, 12:301-310.

11. Pace NR, Thomas BC, Woese CR: Probing RNA structure, function, and history by comparative analysis. The RNA World Cold Spring Harbor Laboratory PressGesteland RF, Cech TR, Atkins JF, 2 1999, 113-141.
12. Xia T, SantaLucia J Jr, Burkard ME, Kierzek R, Schroeder SJ, Jiao X, Cox C, Turner DH: Thermodynamic parameters for an expanded nearestneighbor model for formation of RNA duplexes with Watson-Crick pairs. Biochemistry 1998, 37:14719-14735.

13. Mathews DH, Sabina J, Zuker M, Turner DH: Expanded sequence dependence of thermodynamic parameters provides improved prediction of RNA secondary structure. J Mol Biol 1999, 288:911-940.

14. Mathews DH, Disney MD, Childs JL, Schroeder SJ, Zuker M, Turner DH: Incorporating chemical modification constraints into a dynamic programming algorithm for prediction of RNA secondary structure. Proc Natl Acad Sci USA 2004, 101:7287-7292.

15. Zuker M, Stiegler P: Optimal computer folding of large RNA sequences using thermodynamics and auxiliary information. Nucleic Acids Res 1981, 9:133-148.

16. Ding $Y$, Chan $C Y$, Lawrence $C E$ : RNA secondary structure prediction by centroids in a Boltzmann weighted ensemble. RNA 2005, 11(8):1157-1166.

17. Ding $Y$, Lawrence CE: A statistical sampling algorithm for RNA secondary structure prediction. Nucleic Acids Res 2003, 31(24):7280-7301.

18. Lu ZJ, Gloor JW, Mathews DH: Improved RNA secondary structure prediction by maximizing expected pair accuracy. RNA 2009, 15:1805-1813.

19. Hamada M, Kiryu H, Sato K, Mituyama T, Asai K: Prediction of RNA secondary structure using generalized centroid estimators. Bioinformatics 2009, 25(4):465-473.

20. Dowell RD, Eddy SR: Evaluation of several lightweight stochastic contextfree grammars for RNA secondary structure prediction. BMC Bioinformatics 2004, 5(1):71.

21. Doshi KJ, Cannone JJ, Cobaugh CW, Gutell RR: Evaluation of the suitability of free-energy minimization using nearest-neighbor energy parameters for RNA secondary structure prediction. BMC Bioinformatics 2004, 5(1):105.

22. Mathews DH: Using an RNA secondary structure partition function to determine confidence in base pairs predicted by free energy minimization. RNA 2004, 10:1178-1190.

23. Mathews $\mathrm{DH}$, Turner $\mathrm{DH}$ : Dynalign: An algorithm for finding the secondary structure common to two RNA sequences. J Mol Biol 2002, 317:191-203.

24. Harmanci AO, Sharma G, Mathews DH: PARTS: Probabilistic Alignment for RNA joinT Secondary structure prediction. Nucleic Acids Res 2008, 36:2406-2417.

25. Havgaard JH, Lyngso RB, Stormo GD, Gorodkin J: Pairwise local structural alignment of RNA sequences with sequence similarity less than $40 \%$. Bioinformatics 2005, 21(9):1815-1824.

26. Hofacker IL, Fekete M, Stadler PF: Secondary structure prediction for aligned RNA sequences. J Mol Biol 2002, 319:1059-1066.

27. Lück R, Steger $G$, Riesner D: Thermodynamic prediction of conserved secondary structure: Application to the RRE element of HIV, the tRNAlike element of CMV and the mRNA of prion protein. J Mol Biol 1996, 258:813-826

28. Duan S, Mathews DH, Turner DH: Interpreting oligonucleotide microarray data to determine RNA secondary structure: application to the $3^{\prime}$ end of Bombyx mori R2 RNA. Biochemistry 2006, 45(32):9819-9832.

29. Deigan KE, Li TW, Mathews DH, Weeks KM: Accurate SHAPE-directed RNA structure determination. Proc Natl Acad Sci USA 2009, 106(1):97-102.

30. Hart JM, Kennedy SD, Mathews DH, Turner DH: NMR-assisted prediction of RNA secondary structure: identification of a probable pseudoknot in the coding region of an R2 retrotransposon. J Am Chem Soc 2008, 130(31):10233-10239.

31. Lu ZJ, Mathews DH: Efficient siRNA selection using hybridization thermodynamics. Nucleic Acids Res 2007, 36:640-647.

32. Lu ZJ, Mathews DH: Fundamental differences in the equilibrium considerations for siRNA and antisense oligodeoxynucleotide design. Nucleic Acids Res 2008, 36:3738-3745.

33. Mathews DH, Burkard ME, Freier SM, Wyatt JR, Turner DH: Predicting oligonucleotide affinity to nucleic acid targets. RNA 1999, 5:1458-1469.

34. Tafer H, Ameres SL, Obernosterer G, Gebeshuber CA, Schroeder R, Martinez J, Hofacker IL: The impact of target site accessibility on the design of effective siRNAs. Nat Biotechnol 2008, 26(5):578-583. 
35. Shao Y, Chan CY, Maliyekkel A, Lawrence CE, Roninson IB, Ding Y: Effect of target secondary structure on RNAi efficiency. RNA 2007, 13(10):1631-1640.

36. Long $D$, Lee $R$, Williams $P$, Chan $C Y$, Ambros V, Ding Y: Potent effect of target structure on microRNA function. Nat Struct Mol Biol 2007, 14(4):287-294.

37. Aguirre-Hernandez R, Hoos HH, Condon A: Computational RNA secondary structure design: empirical complexity and improved methods. BMC Bioinformatics 2007, 8:34

38. Flamm C, Hofacker IL, Maurer-Stroh S, Stadler PF, Zehl M: Design of multistable RNA molecules. RNA 2001, 7:254-265.

39. Watts JM, Dang KK, Gorelick RJ, Leonard CW, Bess JW Jr, Swanstrom R, Burch CL, Weeks KM: Architecture and secondary structure of an entire HIV-1 RNA genome. Nature 2009, 460(7256):711-716.

40. Washietl S, Hofacker IL, Stadler PF: Fast and reliable prediction of noncoding RNAs. Proc Natl Acad Sci USA 2005, 102(7):2454-2459.

41. Uzilov AV, Keegan JM, Mathews DH: Detection of non-coding RNAs on the basis of predicted secondary structure formation free energy change. BMC Bioinformatics 2006, 7(1):173.

42. Torarinsson E, Sawera M, Havgaard JH, Fredholm M, Gorodkin J: Thousands of corresponding human and mouse genomic regions unalignable in primary sequence contain common RNA structure. Genome Res 2006, 16(7):885-889.

43. Mathews DH, Andre TC, Kim J, Turner DH, Zuker M: An updated recursive algorithm for RNA secondary structure prediction with improved thermodynamic parameters. Molecular Modeling of Nucleic Acids American Chemical SocietyLeontis NB, SantaLucia J Jr 1998, 246-257.

44. Zuker M: On finding all suboptimal foldings of an RNA molecule. Science 1989, 244:48-52.

45. Steger G, Hofmann H, Fortsch J, Gross HJ, Randles JW, Sanger HL, Riesner D: Conformational transitions in viroids and virusoids: Comparison of results from energy minimization algorithm and from experimental data. J Biomol Struct Dyn 1984, 2(3):543-571.

46. Harmanci AO, Sharma G, Mathews DH: Efficient pairwise RNA structure prediction using probabilistic alignment constraints in Dynalign. $B M C$ Bioinformatics 2007, 8:130.

47. Wuchty S, Fontana W, Hofacker IL, Schuster P: Complete suboptimal folding of RNA and the stability of secondary structures. Biopolymers 1999, 49:145-165.

48. Harmanci AO, Sharma G, Mathews DH: Stochastic sampling of the RNA structural alignment space. Nucleic Acids Res 2009, 37:4063-4075.

49. Smit S, Rother K, Heringa J, Knight R: From knotted to nested RNA structures: a variety of computational methods for pseudoknot removal. RNA 2008, 14(3):410-416.

50. Mathews DH: RNA secondary structure analysis using RNAstructure. Current Protocols in Bioinformatics New York: John Wiley and Sons, IncBaxevanis AD, Davison DB, Page RDM, Petsko GA, Stein LD, Stormo GD 2006, 12.16.11-12.16.14

51. Mathews DH: Predicting the secondary structure common to two sequences with Dynalign. Current Protocols in Bioinformatics New York: John Wiley and Sons, IncBaxevanis AD, Davison DB, Page RDM, Petsko GA, Stein LD, Stormo GD 2005, 12.14.11-12.14.11.

52. Mathews DH, Turner DH, Zuker M: RNA secondary structure prediction. Current Protocols in Nucleic Acid Chemistry New York: John Wiley and SonsBeaucage SL, Bergstrum DE, Glick GD, Jones RA 2000, 11:2.1-2.10.

53. Lu ZJ, Mathews DH: OligoWalk: An online siRNA design tool utilizing hybridization thermodynamics. Nucleic Acids Res 2008, 36:W104-W108.

54. Lu ZJ, Turner DH, Mathews DH: A set of nearest neighbor parameters for predicting the enthalpy change of RNA secondary structure formation. Nucleic Acids Res 2006, 34:4912-4924.

55. Matveeva OV, Mathews DH, Tsodikov AD, Shabalina SA, Gesteland RF, Atkins JF, Freier SM: Thermodynamic criteria for high hit rate antisense oligonucleotide design. Nucleic Acids Res 2003, 31(17):4989-4994.

56. Allawi HT, SantaLucia J Jr: Thermodynamics and NMR of internal GTT mismatches in DNA. Biochemistry 1997, 36(34):10581-10594.

57. Allawi HT, SantaLucia J Jr: NMR solution structure of a DNA dodecamer containing single GT mismatches. Nucleic Acids Res 1998, 26(21):4925-4934.

58. Allawi HT, SantaLucia J Jr: Nearest-neighbor thermodynamics of internal $\mathrm{A} \cdot \mathrm{C}$ mismatches in DNA: sequence dependence and $\mathrm{pH}$ effects. Biochemistry 1998, 37(26):9435-9444.
59. Allawi HT, SantaLucia J Jr: Thermodynamics of internal C.T mismatches in DNA. Nucleic Acids Res 1998, 26(11):2694-2701.

60. Allawi HT, SantaLucia J Jr: Nearest neighbor thermodynamic parameters for internal G'A mismatches in DNA. Biochemistry 1998, 37(8):2170-2179.

61. Peyret N, Seneviratne PA, Allawi HT, SantaLucia J Jr: Nearest-neighbor thermodynamics and NMR of DNA sequences with internal $A \cdot A, C \cdot C, G \cdot G$, and TT mismatches. Biochemistry 1999, 38:3468-3477.

62. Plum GE, Grollman AP, Johnson F, Breslauer KJ: Influence of the oxidatively damaged adduct 8-oxodeoxyguanosine on the conformation, energetics, and thermodynamic stability of a DNA duplex. Biochemistry 1995, 34(49):16148-16160.

63. Bolewska K, Zielenkiewicz A, Wierzchowski KL: Deoxydodecanucleotide heteroduplex d(TTTATAATAAA). d(TTATTATAAAA) containing the promoter Pribnow sequence TATAAT. I. Double-helix stability by UV spectrophotometry and calorimetry. Nucleic Acids Res 1984, 12(7):3245-3256

64. Breslauer KJ, Frank R, Blocker H, Marky LA: Predicting DNA duplex stability from the base sequence. Proc Natl Acad Sci USA 1986, 83(11):3746-3750.

65. Leonard GA, Thomson J, Watson WP, Brown T: High-resolution structure of a mutagenic lesion in DNA. Proc Natl Acad Sci USA 1990, 87(24):9573-9576.

66. Tibanyenda N, De Bruin SH, Haasnoot CAG, Marel Van Der GA, Van Boom JH, Hilbers CW: The effect of single base-pair mismatches on the duplex stability of d(T-A-T-T-A-A-T-A-T-C-A-A-G-T-T-G) $\cdot d(C-A-A-C-T-T-G-$ A-T-A-T-T-A-A-T-A). Eur J Biochem 1984, 139:19-27.

67. Plum GE, Grollman AP, Johnson F, Breslauer KJ: Influence of an exocyclic guanine adduct on the thermal stability, conformation, and melting thermodynamics of a DNA duplex. Biochemistry 1992, 31(48):12096-12102.

68. Arnold FH, Wolk S, Cruz P, Tinoco I Jr: Structure, dynamics, and thermodynamics of mismatched DNA oligonucleotide duplexes $d$ (CCCAGGG)2 and d(CCCTGGG)2. Biochemistry 1987, 26(13):4068-4075.

69. Ratmeyer L, Vinayak R, Zhong YY, Zon G, Wilson WD: Sequence specific thermodynamic and structural properties for DNARNA duplexes. Biochemistry 1994, 33(17):5298-5304

70. Williams AP, Longfellow CE, Freier SM, Kierzek R, Turner DH: Laser temperature-jump, spectroscopic, and thermodynamic study of salt effects on duplex formation by dGCATGC. Biochemistry 1989, 28(10):4283-4291.

71. Sugimoto N, Honda K, Sasaki M: Application of the thermodynamic parameters of DNA stability prediction to double-helix formation of deoxyribooligonucleotides. Necleosides \& Nucleotides 1994, 13:1311-1317.

72. Wu P, Nakano S, Sugimoto N: Temperature dependence of thermodynamic properties for DNA/DNA and RNA/DNA duplex formation. Eur J Biochem 2002, 269(12):2821-2830.

73. Hall KB, McLaughlin LW: Thermodynamic and structural properties of pentamer DNA.DNA, RNA.RNA, and DNA.RNA duplexes of identical sequence. Biochemistry 1991, 30(44):10606-10613.

74. Sugimoto N, Nakano S, Yoneyama M, Honda K: Improved thermodynamic parameters and helix initiation factor to predict stability of DNA duplexes. Nucleic Acids Res 1996, 24(22):4501-4505.

75. Bommarito S, Peyret N, SantaLucia J Jr: Thermodynamic parameters for DNA sequences with dangling ends. Nucleic Acids Res 2000, 28(9):1929-1934.

76. Senior $\mathrm{M}$, Jones RA, Breslauer $\mathrm{KJ}$ : Influence of dangling thymidine residues on the stability and structure of two DNA duplexes. Biochemistry 1988, 27(10):3879-3885.

77. Aboul-ela F, Koh D, Tinoco I Jr, Martin FH: Base-base mismatches. Thermodynamics of double helix formation for dCA3XA3G + dCT3YT3G (X, Y = A, C, G, T). Nucleic Acids Res 1985, 13(13):4811-4824.

78. Riccelli PV, Hilario J, Gallo FJ, Young AP, Benight AS: DNA and RNA oligomer sequences from the $3^{\prime}$ noncoding region of the chicken glutamine synthetase gene from intramolecular hairpins. Biochemistry 1996, 35(48):15364-15372.

79. Rentzeperis D, Alessi K, Marky LA: Thermodynamics of DNA hairpins: contribution of loop size to hairpin stability and ethidium binding. Nucleic Acids Res 1993, 21(11):2683-2689.

80. Vallone PM, Paner TM, Hilario J, Lane MJ, Faldasz BD, Benight AS: Melting studies of short DNA hairpins: influence of loop sequence and adjoining base pair identity on hairpin thermodynamic stability. Biopolymers 1999, 50(4):425-442. 
81. Hilbers CW, Haasnoot CA, de Bruin SH, Joordens JJ, Marel van der GA, van Boom JH: Hairpin formation in synthetic oligonucleotides. Biochimie 1985, 67(7-8):685-695.

82. Antao VP, Tinoco I Jr: Thermodynamic parameters for loop formation in RNA and DNA hairpin tetraloops. Nucleic Acids Res 1992, 20:819-824.

83. Antao VP, Lai SY, Tinoco I Jr: A thermodynamic study of unusually stable RNA and DNA hairpins. Nucleic Acids Res 1991, 19:5901-5905.

84. Moody EM, Bevilacqua PC: Thermodynamic coupling of the loop and stem in unusually stable DNA hairpins closed by CG base pairs. J Am Chem Soc 2003, 125(8):2032-2033.

85. Nakano M, Moody EM, Liang J, Bevilacqua PC: Selection for thermodynamically stable DNA tetraloops using temperature gradient gel electrophoresis reveals four motifs: $d(c G N N A g), d(c G N A B g), d$ (cCNNGg), and d(gCNNGc). Biochemistry 2002, 41(48):14281-14292.

86. LeBlanc DA, Morden KM: Thermodynamic characterization of deoxyribooligonucleotide duplexes containing bulges. Biochemistry 1991, 30(16):4042-4047.

87. Morden $\mathrm{KM}$, Chu YG, Martin FH, Tinoco I Jr: Unpaired cytosine in the deoxynucleotide duplex $\mathrm{dCA}_{3} \mathrm{CA}_{3} \mathrm{GdCT}_{6} \mathrm{G}$ is outside of the helix. Biochemistry 1983, 22:5557-5563.

88. Li Y, Zon G, Wilson WD: Thermodynamics of DNA duplexes with adjacent G.A mismatches. Biochemistry 1991, 30(30):7566-7572.

89. Li Y, Agrawal S: Oligonucleotides containing G'A pairs: effect of flanking sequences on structure and stability. Biochemistry 1995, 34(31):10056-10062.

90. Kadrmas JL, Ravin AJ, Leontis NB: Relative stabilities of DNA three-way, four-way and five-way junctions (multi-helix junction loops): unpaired nucleotides can be stabilizing or destabilizing. Nucleic Acids Res 1995, 23:2212-2222.

91. Leontis NB, Kwok W, Newman JS: Stability and structure of three-way DNA junctions containing unpaired nucleotides. Nucleic Acids Res 1991, 19:759-766.

92. Ladbury JE, Sturtevant JM, Leontis NB: The thermodynamics of formation of a three-strand, DNA three-way junction complex. Biochemistry 1994, 33(22):6828-6833.

93. Sugimoto N, Nakano S, Katoh M, Matsumura A, Nakamuta H, Ohmichi T, Yoneyama M, Sasaki M: Thermodynamic parameters to predict stability of RNA/DNA hybrid duplexes. Biochemistry 1995, 34:11211-11216.

94. Szymanski M, Barciszewska MZ, Barciszewski J, Erdmann VA: 5 S ribosomal RNA database Y2K. Nucleic Acids Res 2000, 28:166-167.

doi:10.1186/1471-2105-11-129

Cite this article as: Reuter and Mathews: RNAstructure: software for RNA secondary structure prediction and analysis. BMC Bioinformatics 2010 11:129.

\section{Submit your next manuscript to BioMed Central and take full advantage of:}

- Convenient online submission

- Thorough peer review

- No space constraints or color figure charges

- Immediate publication on acceptance

- Inclusion in PubMed, CAS, Scopus and Google Scholar

- Research which is freely available for redistribution 